PROCEEDINGS OF THE

AMERICAN MATHEMATICAL SOCIETY

Volume 140, Number 4, April 2012, Pages 1207-1219

S 0002-9939(2011)10989-4

Article electronically published on August 2, 2011

\title{
ON OPEN AND CLOSED MORPHISMS BETWEEN SEMIALGEBRAIC SETS
}

\author{
JOSÉ F. FERNANDO AND J. M. GAMBOA
}

(Communicated by Lev Borisov)

\begin{abstract}
In this work we study how open and closed semialgebraic maps between two semialgebraic sets extend, via the corresponding spectral maps, to the Zariski and maximal spectra of their respective rings of semialgebraic and bounded semialgebraic functions.
\end{abstract}

\section{INTRODUCTION}

A subset $M \subset \mathbb{R}^{n}$ is said to be basic semialgebraic if it can be written as

$$
M=\left\{x \in \mathbb{R}^{n}: f(x)=0, g_{1}(x)>0, \ldots, g_{m}(x)>0\right\}
$$

for some polynomials $f, g_{1}, \ldots, g_{m} \in \mathbb{R}\left[\mathrm{x}_{1}, \ldots, \mathrm{x}_{n}\right]$. The finite unions of basic semialgebraic sets are called semialgebraic sets. A continuous function $f: M \rightarrow \mathbb{R}$ is said to be semialgebraic if its graph is a semialgebraic subset of $\mathbb{R}^{n+1}$. Usually, semialgebraic function just means a function, not necessarily continuous, whose graph is semialgebraic. However, since all semialgebraic functions occurring in this article are continuous, we will omit for simplicity the continuity condition when we refer to them.

The sum and product of functions, defined pointwise, endow the set $\mathcal{S}(M)$ of semialgebraic functions on $M$ with a natural structure of commutative ring whose unity is the semialgebraic function with constant value 1 . In fact $\mathcal{S}(M)$ is an $\mathbb{R}$ algebra if we identify each real number $r$ with the constant function which just attains this value. The most simple examples of semialgebraic functions on $M$ are the restrictions to $M$ of polynomials in $n$ variables. Other relevant ones are the absolute value of a semialgebraic function, the maximum and the minimum of a finite family of semialgebraic functions, the inverse and the $k$-root of a semialgebraic function whenever these operations are well-defined.

It is obvious that the subset $\mathcal{S}^{*}(M)$ of bounded semialgebraic functions on $M$ is a real subalgebra of $\mathcal{S}(M)$. In what follows, we denote by $\mathcal{S}^{\diamond}(M)$, indistinctly, either $\mathcal{S}(M)$ or $\mathcal{S}^{*}(M)$ in case the involved statements or arguments are valid for both rings. Moreover, if $p \in M$, we will denote by $\mathfrak{m}_{p}^{\diamond}$ the maximal ideal of all functions in $\mathcal{S}^{\diamond}(M)$ vanishing at $p$.

Received by the editors July 26, 2010 and, in revised form, January 3, 2011.

2010 Mathematics Subject Classification. Primary 14P10, 54C30; Secondary 12D15, 13E99.

Key words and phrases. Semialgebraic function, semialgebraic set, Zariski spectrum, maximal spectrum, open and closed maps, proper map, Bezoutian, quotient map.

The authors were supported by the Spanish GAAR MTM2008-00272, Proyecto Santander Complutense PR34/07-15813 and GAAR Grupos UCM 910444. 
Likewise, a semialgebraic map $\varphi: N \rightarrow M$ between semialgebraic sets $N \subset \mathbb{R}^{n}$ and $M \subset \mathbb{R}^{m}$ is a continuous map whose coordinates are semialgebraic functions.

Given an open and closed map between two semialgebraic sets, our purpose is to study the topological properties of its unique continuous extensions to the Zariski and maximal spectra of their respective rings of semialgebraic and bounded semialgebraic functions.

(1.1) Since the usual notation for spectral spaces becomes cumbersome, we replace it by the following notation. Let $M \subset \mathbb{R}^{n}$ be a semialgebraic set. We denote

$$
\begin{aligned}
& \operatorname{Spec}_{\mathrm{s}}(M)=\operatorname{Spec}(\mathcal{S}(M)) ; \\
& \operatorname{Spec}_{\mathrm{S}}^{*}(M)=\operatorname{Spec}\left(\mathcal{S}^{*}(M)\right) ; \\
& \operatorname{Spec}_{\mathrm{s}}^{\diamond}(M)=\operatorname{Spec}\left(\mathcal{S}^{\diamond}(M)\right), \\
& \beta_{\mathrm{s}} M=\operatorname{Spec}_{\text {max }}(\mathcal{S}(M)) ; \\
& \beta_{\mathrm{s}}^{*}=\operatorname{Spec}_{\text {max }}\left(\mathcal{S}^{*}(M)\right) ; \\
& \beta_{\mathrm{s}}^{\diamond}=\operatorname{Spec}_{\text {max }}\left(\mathcal{S}^{\diamond}(M)\right) .
\end{aligned}
$$

All these spaces are endowed with the Zariski topology; see (2.1) below. Recall, FG4, 3.5], that the map $\Phi_{M}: \beta_{\mathrm{s}} M \rightarrow \beta_{\mathrm{s}}^{*} M, \mathfrak{m} \mapsto \mathfrak{m}^{*}$, where $\mathfrak{m}^{*}$ is the unique maximal ideal of $\mathcal{S}^{*}(M)$ containing the prime ideal $\mathfrak{m} \cap \mathcal{S}^{*}(M)$, is a homeomorphism. On the other hand, the map

$$
\phi: M \rightarrow \operatorname{Spec}_{\mathrm{s}}^{\diamond}(M), p \mapsto \mathfrak{m}_{p}^{\diamond}
$$

embeds $M$ (endowed with the Euclidean topology) into $\operatorname{Spec}_{\mathrm{s}}^{\diamond}(M)$ as a dense subspace. In fact we identify $M \equiv \phi(M) \subset \beta_{\mathrm{s}}^{\diamond} M$ and denote $\partial M=\beta_{\mathrm{s}}^{*} M \backslash M$.

(1.2) Each semialgebraic map $\varphi: N \rightarrow M$ between semialgebraic sets $N \subset \mathbb{R}^{n}$ and $M \subset \mathbb{R}^{m}$ induces a ring homomorphism $\varphi^{\diamond, \star}: \mathcal{S}^{\diamond}(M) \rightarrow \mathcal{S}^{\diamond}(N), f \mapsto f \circ \varphi$, and the spectral map associated to $\varphi$ is $\operatorname{Spec}_{\mathrm{S}}^{\diamond}(\varphi): \operatorname{Spec}_{\mathrm{S}}^{\diamond}(N) \rightarrow \operatorname{Spec}_{\mathrm{S}}^{\diamond}(M), \mathfrak{p} \mapsto$ $\left(\varphi^{\diamond, \star}\right)^{-1}(\mathfrak{p})$. In fact, $\operatorname{Spec}_{\mathrm{S}}^{\diamond}(\varphi)$ is the unique continuous extension of $\varphi$ with values in $\operatorname{Spec}_{\mathrm{s}}^{\diamond}(M)$, because $N$ is dense in $\operatorname{Spec}_{\mathrm{s}}^{\diamond}(N)$. Moreover, we proved in [FG2, 5.9] that $\operatorname{Spec}_{\mathrm{S}}^{*}(\varphi): \operatorname{Spec}_{\mathrm{s}}^{*}(N) \rightarrow \operatorname{Spec}_{\mathrm{s}}^{*}(M)$ maps $\beta_{\mathrm{s}}^{*} N$ into $\beta_{\mathrm{s}}^{*} M$; we denote $\beta_{\mathrm{s}}^{*} \varphi=$ $\left.\operatorname{Spec}_{\mathrm{s}}^{*}(\varphi)\right|_{\beta_{\mathrm{s}}^{*} N}: \beta_{\mathrm{s}}^{*} N \rightarrow \beta_{\mathrm{s}}^{*} M$.

(1.3) On the other hand, we proved in [FG2, 4.8] the existence of semialgebraic maps $\varphi: N \rightarrow M$ and maximal ideals of $\mathcal{S}(N)$ whose image under the induced

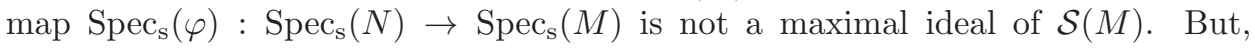
$\mathcal{S}(M)$ being a Gelfand ring (see [FG1, 3.1(iii)] for an elementary proof), the map $\mathbf{s}_{M}: \operatorname{Spec}_{\mathrm{s}}(M) \rightarrow \beta_{\mathrm{s}} M$, which maps each prime ideal of $\mathcal{S}(M)$ to the unique maximal ideal of $\mathcal{S}(M)$ containing it, is, by [MO, 1.2], a (continuous) retraction. We define $\beta_{\mathrm{s}} \varphi=\left.\mathrm{s}_{M} \circ \operatorname{Spec}_{\mathrm{S}}(\varphi)\right|_{\beta_{\mathrm{s}} N}: \beta_{\mathrm{s}} N \rightarrow \beta_{\mathrm{s}} M$, which is a continuous map. Note that $N$ and $M$ being dense in $\beta_{\mathrm{s}} N$ and $\beta_{\mathrm{s}} M$ respectively, the map $\beta_{\mathrm{s}} \varphi$ is the unique continuous extension of $\varphi: N \rightarrow M$ to $\beta_{\mathrm{s}} N$ taking values in $\beta_{\mathrm{s}} M$.

(1.4) Again by [FG1, 3.1(iii)] and [MO, 1.2], there exists a retraction $r_{M}$ : $\operatorname{Spec}_{\mathrm{s}}^{*}(M) \rightarrow \beta_{\mathrm{s}}^{*} M$ which maps each prime ideal of $\mathcal{S}^{*}(M)$ to the unique maximal ideal of $\mathcal{S}^{*}(M)$ containing it. Consider the inclusion maps $i_{M}: \beta_{\mathrm{s}} M \hookrightarrow \operatorname{Spec}_{\mathrm{S}}(M)$ and $j_{M}: \beta_{\mathrm{s}}^{*} M \hookrightarrow \operatorname{Spec}_{\mathrm{S}}^{*}(M)$, and let $k_{M}: \operatorname{Spec}_{\mathrm{s}}(M) \rightarrow \operatorname{Spec}_{\mathrm{S}}^{*}(M), \mathfrak{p} \mapsto \mathfrak{p} \cap \mathcal{S}^{*}(M)$, which is a homeomorphism onto its image; see [FG2, 3.2]. Moreover, $\mathbf{s}_{M}=$ $\Phi_{M}^{-1} \circ \mathrm{r}_{M} \circ k_{M}$ and $k_{M} \circ \operatorname{Spec}_{\mathrm{s}}(\varphi)=\operatorname{Spec}_{\mathrm{S}}^{*}(\varphi) \circ k_{N}$. This, together with the equality $r_{M} \circ \operatorname{Spec}_{\mathrm{S}}^{*}(\varphi) \circ j_{N}=\operatorname{Spec}_{\mathrm{S}}^{*}(\varphi) \circ j_{N}$, provides the following commutative 
diagram:

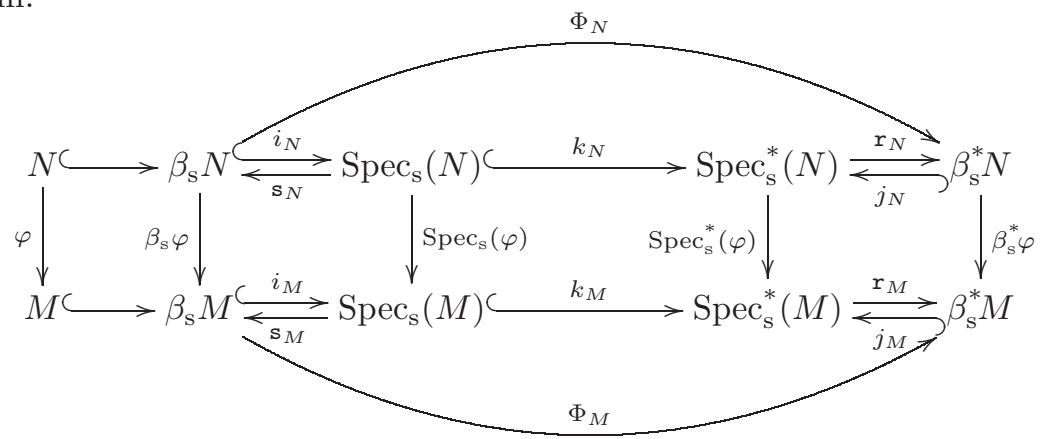

Thus, via $\Phi_{N}$ and $\Phi_{M}$, we can translate the properties of the operator $\beta_{\mathrm{s}}^{*}$ to properties of $\beta_{\mathrm{s}}$. This is why we focus our attention on the study of the behaviour of $\beta_{\mathrm{s}}^{*}$.

As one can imagine, to get relevant information about $\operatorname{Spec}_{\mathrm{s}}^{*}(\varphi)$ and its restriction $\beta_{\mathrm{s}}^{*} \varphi$ we must impose strong conditions to the map $\varphi$. Moreover, by the nature of the used techniques, which come back to $\underline{\mathrm{P}}$ (see also $\mathrm{Mu}$ ), we restrict ourselves to maps $\varphi$ which are bounded over their fibers. This is why in dealing with not necessarily bounded semialgebraic functions, we impose $\varphi$ to be a proper map. Our main results in this direction are the following:

Theorem 1.5. Let $\varphi: N \rightarrow M$ be an open, closed and surjective semialgebraic map. Then both maps $\operatorname{Spec}_{\mathrm{s}}^{*}(\varphi): \operatorname{Spec}_{\mathrm{s}}^{*}(N) \rightarrow \operatorname{Spec}_{\mathrm{s}}^{*}(M)$ and $\beta_{\mathrm{s}}^{*} \varphi: \beta_{\mathrm{s}}^{*} N \rightarrow \beta_{\mathrm{s}}^{*} M$ are open, proper and surjective.

Theorem 1.6. Let $\varphi: N \rightarrow M$ be a semialgebraic map. Then the following assertions are equivalent:

(i) The $\operatorname{map} \varphi: N \rightarrow M$ is open, proper and surjective.

(ii) The map $\operatorname{Spec}_{\mathrm{s}}^{*}(\varphi): \operatorname{Spec}_{\mathrm{s}}^{*}(N) \rightarrow \operatorname{Spec}_{\mathrm{s}}^{*}(M)$ is open, proper and surjective and $\beta_{\mathrm{s}}^{*} \varphi(\partial N)=\partial M$.

(iii) The map $\operatorname{Spec}_{\mathrm{s}}(\varphi): \operatorname{Spec}_{\mathrm{s}}(N) \rightarrow \operatorname{Spec}_{\mathrm{s}}(M)$ is open, proper and surjective and the fiber under $\varphi$ of each isolated point of $M$ is compact.

(iv) The $\operatorname{map} \beta_{\mathrm{s}}^{*} \varphi: \beta_{\mathrm{s}}^{*} N \rightarrow \beta_{\mathrm{s}}^{*} M$ is open, proper and surjective and $\beta_{\mathrm{s}}^{*} \varphi(\partial N)=$ $\partial M$.

A source of examples of maps to which Theorems 1.5 and 1.6 apply is the unramified semialgebraic coverings with finite fibers. However, other maps fit such a situation.

Examples 1.7. (i) Let $N=\left\{\left(x^{2}-y^{2}-z^{2}\right)\left(x^{2}-y^{2}+z^{2}\right)=0\right\} \subset \mathbb{R}^{3}, M=\mathbb{R}^{2}$ and $\varphi$ be the restriction to $N$ of the projection $\mathbb{R}^{3} \rightarrow \mathbb{R}^{2},(x, y, z) \rightarrow(x, y)$. One can check that $\varphi$ is open, proper and surjective.

(ii) Let $N_{1}, \ldots, N_{r} \subset \mathbb{R}^{n}$ be a finite collection of compact semialgebraic sets and let $\varphi_{i}: N_{1} \times \cdots \times N_{r} \rightarrow N_{i},\left(x_{1}, \ldots, x_{r}\right) \mapsto x_{i}$ be the projection onto the $i$ th-factor. Then $\varphi_{i}$ is open, proper and surjective.

(iii) The symmetric group $\mathcal{S}_{n}$ acts in a natural way on $\mathbb{R}^{n}$. The space of orbits $\mathbb{R}^{n} / \mathcal{S}_{n}$ admits a natural structure of affine semialgebraic space (see $[\mathrm{B}, 1.6]$ ) and it is homeomorphic to the semialgebraic subset $M=\left\{x \in \mathbb{R}^{n}: \operatorname{Bez}(x)\right.$ is positive semidefinite $\}$, where Bez denotes the quadratic form usually known as Bezoutian (see [BCR, 6.2.7] and [PS, 0.1]). In fact, the map $\sigma: \mathbb{R}^{n} \rightarrow \mathbb{R}^{n}, x \mapsto\left(\sigma_{1}(x), \ldots, \sigma_{n}(x)\right)$, 
where $\sigma_{1}, \ldots, \sigma_{n}$ are the elementary symmetric functions, induces a homeomorphism between $\mathbb{R}^{n} / \mathcal{S}_{n}$ and $M$. In fact, the semialgebraic map $\sigma: \mathbb{R}^{n} \rightarrow M$ is open, proper and surjective.

(iv) In general, if a finite group of semialgebraic automorphisms acts on $\mathbb{R}^{n}$, we get a "semialgebraic" quotient (see [B, 1.6]) and the canonical projection is a "semialgebraic" map which is moreover open, proper and surjective.

The article is organized as follows. In Section 2 we present some preliminary results concerning Zariski spectra of rings of semialgebraic and bounded semialgebraic functions on a semialgebraic set that will be useful to prove Theorems 1.5] and 1.6 given in Section 3 .

\section{Preliminaries on Zariski AND MAXimal SPECTRA}

We devote this section to recalling the main properties of the Zariski spectra of rings of semialgebraic and bounded semialgebraic functions on a semialgebraic set that we need in the sequel (see for instance [FG2, §3- $\$ 6]$ for further details) and the notation to be used.

(2.1) Zariski spectra of rings of semialgebraic functions. Recall that the Zariski spectrum $\operatorname{Spec}_{\mathrm{s}}^{\diamond}(M)$ of $\mathcal{S}^{\diamond}(M)$ is the collection of all prime ideals of $\mathcal{S}^{\diamond}(M)$. This set $\operatorname{Spec}_{\mathrm{S}}^{\diamond}(M)$ is endowed with the Zariski topology which has as a basis of open sets the family of sets $\mathcal{D}_{\operatorname{Spec}_{\mathrm{s}}^{\diamond}(M)}(f)=\left\{\mathfrak{p} \in \operatorname{Spec}_{\mathrm{S}}^{\diamond}(M): f \notin \mathfrak{p}\right\}$ where $f \in \mathcal{S}^{\diamond}(M)$. We will denote its complement by $z_{\operatorname{Spec}_{\mathrm{s}}^{\diamond}(M)}(f)=\operatorname{Spec}_{\mathrm{s}}^{\diamond}(M) \backslash \mathcal{D}_{\operatorname{Spec}_{\mathrm{s}}^{\diamond}(M)}(f)$.

More generally, for each ideal $\mathfrak{a}$ of a commutative ring with unity $R$, we denote $Z_{\operatorname{Spec}(R)}(\mathfrak{a})=\{\mathfrak{p} \in \operatorname{Spec}(R): \mathfrak{a} \subset \mathfrak{p}\}$. If $\mathfrak{a}=a R$ is a principal ideal, we write $z_{\operatorname{Spec}(R)}(a)=z_{\operatorname{Spec}(R)}(\mathfrak{a})$. Next we recall some standard notation. If $\psi: A \rightarrow B$ is a ring homomorphism and $\mathfrak{p} \in \operatorname{Spec}(A)$, we identify

$$
\operatorname{Spec}\left(B_{\mathfrak{p}}\right)=\{\mathfrak{q} \in \operatorname{Spec}(B): \mathfrak{q} \subset \psi(\mathfrak{p}) B\} .
$$

If $\psi$ is moreover injective, we write $\mathfrak{b} \cap A=\psi^{-1}(\mathfrak{b})$ for each ideal $\mathfrak{b}$ in $B$, and $\mathfrak{a} B$ for the smallest ideal of $B$ containing $\psi(\mathfrak{a})$.

[2.1]1) It is well known (see [FG2, §3] for an elementary proof) that the Zariski and the real spectrum of $\mathcal{S}^{\diamond}(M)$ coincide. Consequently, the subset $\beta_{\mathrm{s}}^{\diamond} M$ of closed points of $\operatorname{Spec}_{\mathrm{s}}^{\diamond}(M)$ is, by [BCR, 7.1.25(ii)], a compact, Hausdorff space which contains $M$ as a dense subspace; that is, $\beta_{\mathrm{s}}^{\diamond} M$ is a Hausdorff compactification of $M$. Observe that if $M$ is compact, then the embedding $\phi: M \rightarrow \beta_{\mathrm{s}}^{\diamond} M, p \mapsto \mathfrak{m}_{p}^{\diamond}$ is in fact bijective (because in this case $M$ is dense and closed in $\beta_{\mathrm{s}}^{\diamond} M$ ) and so $\beta_{\mathrm{s}}^{\diamond} M \equiv M$. We denote

$$
\mathcal{D}_{\beta_{\mathrm{s}}^{\diamond} M}(f)=\mathcal{D}_{\operatorname{Spec}_{\mathrm{s}}^{\diamond}(M)}(f) \cap \beta_{\mathrm{s}}^{\diamond} M
$$

and

$$
\mathcal{Z}_{\beta_{\mathrm{s}}^{\diamond} M}(f)=\beta_{\mathrm{s}}^{\diamond} \backslash \backslash \mathcal{D}_{\beta_{\mathrm{s}}^{\diamond} M}(f)=Z_{\operatorname{Spec}_{\mathrm{s}}^{\diamond}(M)}(f) \cap \beta_{\mathrm{s}}^{\diamond} M
$$

(2.12) As usual, given $f, g \in \mathcal{S}^{\diamond}(M)$, we say that $f \leq g$ if $f(x) \leq g(x)$ for all $x \in M$; in the same vein, $f$ is nonnegative if $f \geq 0$. Moreover, the prime ideals of the ring $\mathcal{S}^{\diamond}(M)$ satisfy a "convexity condition" which is ubiquitous in real geometry. Namely, given $f, g \in \mathcal{S}^{\diamond}(M)$ such that $g \in \mathfrak{p}$ and $0 \leq f \leq g$, then also $f \in \mathfrak{p}$ (see FG2, 3.1.2]).

(2.13) A useful consequence of the convexity is the following: The set of prime ideals of the ring $\mathcal{S}^{\diamond}(M)$ containing a fixed prime ideal $\mathfrak{p}$ forms a chain. 


\section{OPEN AND CLOSED SEMIALGEBRAIC MORPHISMS}

The purpose of this section is to prove Theorems 1.5 and 1.6. First, we proceed to develop some auxiliary results to prove Theorem 1.5. To simplify the statements we fix a surjective closed and open semialgebraic map $\varphi: N \rightarrow M$ and denote by $\varphi^{\star}: \mathcal{S}^{*}(M) \hookrightarrow \mathcal{S}^{*}(N), f \mapsto f \circ \varphi$ the induced injective homomorphism. Notice that, since $\varphi$ is open and surjective, the image of $\varphi^{\star}$ consists of those functions in $\mathcal{S}^{*}(N)$ which are constant on the fibers of $\varphi$.

Since $\varphi^{\star}$ is injective, we will write $f$ instead of $\varphi^{\star}(f)$ for each $f \in \mathcal{S}^{*}(M)$, and in this way we identify $\mathcal{S}^{*}(M)$ with the subring of $\mathcal{S}^{*}(N)$ consisting of those $f \in \mathcal{S}^{*}(N)$ which are constant on the fibers of $\varphi$.

We consider in $\mathcal{S}^{*}(N)$ the $\mathcal{S}^{*}(M)$-module structure induced by $\varphi^{\star}$, and for each prime ideal $\mathfrak{p}$ of $\mathcal{S}^{*}(M)$ we denote by $\mathcal{S}^{*}(N)_{\mathfrak{p}}$ the localization of $\mathcal{S}^{*}(N)$ at the multiplicatively closed set $\mathcal{S}^{*}(M) \backslash \mathfrak{p}$. As usual, given functions $f \in \mathcal{S}^{*}(M)$ and $g \in \mathcal{S}^{*}(N)$ we will write $f g=\varphi^{\star}(f) g \in \mathcal{S}^{*}(N)$.

Next, we present an elementary but useful construction, which originates in $[\mathrm{P}]$.

Construction 3.1. Let $\varphi: N \rightarrow M$ be an open, closed and surjective semialgebraic map. Let $h \in \mathcal{S}^{*}(N)$. Then, the functions

$$
h_{+}: M \rightarrow \mathbb{R}, y \mapsto \sup \{h(x): \varphi(x)=y\}
$$

and

$$
h_{-}: M \rightarrow \mathbb{R}, y \mapsto \inf \{h(x): \varphi(x)=y\}
$$

are bounded and semialgebraic. Indeed, since $h_{-}=-(-h)_{+}$, it is sufficient to study the function $h_{+}$, which is bounded because $h$ is. As to the continuity, and since $\varphi$ is open and surjective, the topology on $M$ is the quotient topology for $\varphi$, and so it suffices to prove the continuity of $f=h_{+} \circ \varphi$. Fix $x_{0} \in N$ and $\varepsilon>0$. Let $x_{1} \in \varphi^{-1}\left(\varphi\left(x_{0}\right)\right)$ such that $f\left(x_{0}\right)-\varepsilon / 2<h\left(x_{1}\right) \leq f\left(x_{0}\right)$ and consider the open intervals $I=\left(-\infty, f\left(x_{0}\right)+\varepsilon / 2\right)$ and $J=\left(f\left(x_{0}\right)-\varepsilon / 2,+\infty\right)$. Observe that the open semialgebraic subsets $U=h^{-1}(I)$ and $V=h^{-1}(J)$ of $N$ contain, respectively, $\varphi^{-1}\left(\varphi\left(x_{0}\right)\right)$ and $x_{1}$. Define $W_{1}=\varphi^{-1}(M \backslash \varphi(N \backslash U))$ and $W_{2}=\varphi^{-1}(\varphi(V))$. Clearly, since $\varphi$ is open and closed, both $W_{1}$ and $W_{2}$ are open semialgebraic subsets of $N$; moreover, $x_{0} \in W=W_{1} \cap W_{2}$ and in fact $\varphi^{-1}(\varphi(x)) \subset U$ for all $x \in W_{1}$. As one can check straightforwardly, these sets fulfill $f\left(W_{1}\right) \subset\left(-\infty, f\left(x_{0}\right)+\varepsilon / 2\right]$ and $f\left(W_{2}\right) \subset\left(f\left(x_{0}\right)-\varepsilon / 2,+\infty\right)$. Thus, $W$ is an open (semialgebraic) neighbourhood of $x_{0}$ in $N$ satisfying $f(W) \subset\left(f\left(x_{0}\right)-\varepsilon, f\left(x_{0}\right)+\varepsilon\right)$, which proves the continuity of $f$ at $x_{0}$.

Finally, notice that the graph of $h_{+}$is a semialgebraic subset of $M \times \mathbb{R}$ because both $\varphi$ and $f$ are semialgebraic and the supremum condition can be expressed in the first order language of the theory of ordered fields.

Remarks 3.2. (i) Given a function $h \in \mathcal{S}^{*}(N)$, we have $h_{-} \circ \varphi \leq h \leq h_{+} \circ \varphi$. Moreover, if $g \in \mathcal{S}^{*}(N)$ is constant on the fibers of $\varphi$, then $g_{-} \circ \varphi=g=g_{+} \circ \varphi$.

(ii) If the functions $h, b \in \mathcal{S}^{*}(N)$ satisfy $h \leq b$, then $h_{-} \leq b_{-}$and $h_{+} \leq b_{+}$.

(iii) For each $f \in \mathcal{S}^{*}(M)$ we have $\varphi^{\star}(|f|)=\left|\varphi^{\star}(f)\right|$, because both functions are nonnegative and share the square:

$$
\left(\varphi^{\star}(|f|)\right)^{2}=\varphi^{\star}\left(|f|^{2}\right)=\varphi^{\star}\left(f^{2}\right)=\left(\left|\varphi^{\star}(f)\right|\right)^{2} .
$$

Thus, the identification $f=\varphi^{\star}(f)$ for $f \in \mathcal{S}^{*}(M)$ is compatible with absolute values. 
(iv) The continuity of the functions $h_{-}$and $h_{+}$fails to be true under milder conditions on the map $\varphi$. Indeed, we can

(1) Consider the closed and surjective semialgebraic map $\varphi: \mathbb{R} \rightarrow \mathbb{R}, t \mapsto t^{3}-3 t$, which is not open because it has a local maximum at $t=-1$. Define $h: \mathbb{R} \rightarrow$ $\mathbb{R}, x \mapsto x /\left(1+x^{2}\right)$. The function $h_{-}: \mathbb{R} \rightarrow \mathbb{R}, y \mapsto \min \left\{h(x): y=x^{3}-3 x\right\}$ is not continuous at $y=2$.

Indeed, for each $y \in \mathbb{R}$ consider the polynomial $P_{y}(\mathrm{x})=\mathrm{x}^{3}-3 \mathrm{x}-y \in \mathbb{R}[\mathrm{x}]$ whose discriminant $\Delta_{y}=27\left(4-y^{2}\right)$ vanishes at $y=2$. In fact $h_{-}(2)=-1 / 2$, because $P_{2}(\mathrm{x})=(\mathrm{x}+1)^{2}(\mathrm{x}-2)$. On the other hand, for every $\varepsilon>0$ one has $\Delta_{y}(2+\varepsilon)<0$, and so the polynomial $P_{2+\varepsilon}$ has a unique real root $\xi_{\varepsilon}$. Thus, $h_{-}(2+\varepsilon)=h\left(\xi_{\varepsilon}\right)=\xi_{\varepsilon} /\left(1+\xi_{\varepsilon}^{2}\right)$. To estimate the value of $\xi_{\varepsilon}$ notice that

$$
P_{2+\varepsilon}(2)=-\varepsilon<0 \quad \text { while } \quad P_{2+\varepsilon}(2+\varepsilon)=\varepsilon(2+\varepsilon)(4+\varepsilon)>0,
$$

and so $2<\xi_{\varepsilon}<2+\varepsilon$. Hence, $\lim _{y \rightarrow 2^{+}} h_{-}(y)=2 / 5 \neq-1 / 2$, and the function $h_{-}$ is not continuous at $y=2$.

(2) Consider the open and surjective semialgebraic map $\varphi: \mathbb{R}^{2} \rightarrow \mathbb{R},(x, y) \mapsto y$ and the semialgebraic function $h: \mathbb{R}^{2} \rightarrow \mathbb{R},(x, y) \rightarrow 1 /\left(1+(x y-1)^{2}\right)$. Notice that

$$
h_{+}: \mathbb{R} \rightarrow \mathbb{R}, y \mapsto \frac{1}{\inf \left\{1+(x y-1)^{2}, x \in \mathbb{R}\right\}}=\left\{\begin{array}{cc}
1 & \text { if } y \neq 0, \\
1 / 2 & \text { if } y=0
\end{array}\right.
$$

is not continuous at $y=0$.

Lemma 3.3. Let $\varphi: N \rightarrow M$ be an open, closed and surjective semialgebraic map. Let $\mathfrak{p}$ be a prime ideal in $\mathcal{S}^{*}(M)$ and let $h \in \mathcal{S}^{*}(N)$ be a nonnegative function whose image under the canonical homomorphism $\mathcal{S}^{*}(N) \rightarrow \mathcal{S}^{*}(N)_{\mathfrak{p}}$ is a unit. Then, also the image of $h_{-}$under the canonical homomorphism $\mathcal{S}^{*}(M) \rightarrow \mathcal{S}^{*}(M)_{\mathfrak{p}}$ is a unit.

Proof. We must prove that $h_{-} \notin \mathfrak{p}$. By the hypothesis on $h$ there exist $g \in \mathcal{S}^{*}(N)$ and $f_{1}, f_{2} \in \mathcal{S}^{*}(M) \backslash \mathfrak{p}$ such that $f_{1} g h=f_{2}$. Since $f_{2} \notin \mathfrak{p}$, the product $f_{1} g$ is not identically zero. Hence, $r=\sup \left\{\left|\left(f_{1} \circ \varphi\right)(x) g(x)\right|: x \in N\right\}>0$. The function $b=\left|f_{1} g\right| / r$ satisfies $0 \leq b(x) \leq 1$ for all $x \in N$ and

$$
H=b h=\frac{\left|f_{1} g h\right|}{r}=\frac{\left|f_{2}\right|}{r} \in \mathcal{S}^{*}(M) .
$$

This last equation means that $H$ is constant on the fibers of $\varphi$. Therefore from the obvious inequalities $0 \leq(H \circ \varphi) \leq h$ on $N$, it follows, by Remark 3.2(ii), that $0 \leq H_{-} \leq h_{-}$on $M$. Now, to prove that $h_{-} \notin \mathfrak{p}$, it suffices, by Remark (2.12), to check that $H_{-} \notin \mathfrak{p}$. But this is clear since, using $3.2(\mathrm{i}), H_{-}=H=\left|f_{2}\right| / r \notin \mathfrak{p}$, and we are done.

Lemma 3.4 (Going-up). Let $\varphi: N \rightarrow M$ be an open, closed and surjective semialgebraic map. Then, the homomorphism $\varphi^{\star}: \mathcal{S}^{*}(M) \rightarrow \mathcal{S}^{*}(N), f \mapsto f \circ \varphi$ satisfies the going-up property.

Proof. Let $\mathfrak{q}$ be a prime ideal in $\mathcal{S}^{*}(N)$, and let $\mathfrak{p}$ be a prime ideal in $\mathcal{S}^{*}(M)$ containing $\mathfrak{q} \cap \mathcal{S}^{*}(M)$. Suppose, by way of contradiction, that no prime ideal of $\mathcal{S}^{*}(N)$ lying over $\mathfrak{p}$ contains $\mathfrak{q}$. Via the canonical homomorphism $\psi: \mathcal{S}^{*}(N) \rightarrow$ $\mathcal{S}^{*}(N)_{\mathfrak{p}}$ we can interpret $\operatorname{Spec}\left(\mathcal{S}^{*}(N)_{\mathfrak{p}}\right)$ as a subset of $\operatorname{Spec}_{\mathrm{S}}^{*}(N)$, and the set of prime ideals of $\mathcal{S}^{*}(N)$ lying over $\mathfrak{p}$ is $Z_{\operatorname{Spec}_{\mathbf{s}}^{*}(N)}\left(\mathfrak{p} \mathcal{S}^{*}(N)\right) \cap \operatorname{Spec}\left(\mathcal{S}^{*}(N)_{\mathfrak{p}}\right)$. Hence, 
our assumption means that if $\mathfrak{a}=\mathfrak{q}+\mathfrak{p} \mathcal{S}^{*}(N)$, then

$$
\begin{aligned}
z_{\operatorname{Spec}_{\mathrm{s}}^{*}(N)}(\mathfrak{a}) \cap \operatorname{Spec}\left(\mathcal{S}^{*}(N)_{\mathfrak{p}}\right) & =z_{\operatorname{Spec}_{\mathrm{s}}^{*}(N)}(\mathfrak{q}) \cap z_{\operatorname{Spec}_{\mathfrak{s}}^{*}(N)}\left(\mathfrak{p} \mathcal{S}^{*}(N)\right) \cap \operatorname{Spec}\left(\mathcal{S}^{*}(N)_{\mathfrak{p}}\right) \\
& =\varnothing .
\end{aligned}
$$

Therefore, $\mathfrak{a} \mathcal{S}^{*}(N)_{\mathfrak{p}}=\mathcal{S}^{*}(N)_{\mathfrak{p}}$, and so there exist $h \in \mathfrak{q}, f_{1}, \ldots, f_{m} \in \mathfrak{p}, g_{1}, \ldots, g_{m} \in$ $\mathcal{S}^{*}(N)$ such that the image under $\psi$ of the function $F=h+f_{1} g_{1}+\cdots+f_{m} g_{m} \in$ $\mathcal{S}^{*}(N)$ is a unit in $\mathcal{S}^{*}(N)_{\mathfrak{p}}$. Hence, $|F| \in \mathcal{S}^{*}(N)$ is a nonnegative function whose image in $\mathcal{S}^{*}(N)_{\mathfrak{p}}$ is a unit.

Let $L$ be a common upper bound for all functions $\left|g_{i}\right|$ and define new functions $\tilde{h}=|h| \in \mathfrak{q}$ and $\tilde{f}=L \sum_{i=1}^{m}\left|f_{i}\right| \in \mathfrak{p}$. By (2.12), and since $0 \leq \tilde{h}_{-} \circ \varphi \leq \tilde{h}$ on $N$, we get $\tilde{h}_{-} \in \mathfrak{q} \cap \mathcal{S}^{*}(M) \subset \mathfrak{p}$, and so $\tilde{h}_{-}+\tilde{f} \in \mathfrak{p}$. Notice that, $\tilde{f}$ being constant on the fibers of $\varphi$, we have $(\tilde{h}+\tilde{f})_{-}=\tilde{h}_{-}+\tilde{f} \in \mathfrak{p}$. On the other hand,

$$
|F| \leq|h|+\sum_{i=1}^{m}\left|g_{i}\right|\left|f_{i}\right| \leq \tilde{h}+L \sum_{i=1}^{m}\left|f_{i}\right|=\tilde{h}+\tilde{f},
$$

on $N$. This, together with the fact that $|F|$ is a unit in $\mathcal{S}^{*}(N)_{\mathfrak{p}}$ implies, by (2.12), that the image of $\tilde{h}+\tilde{f}$ in $\mathcal{S}^{*}(N)_{\mathfrak{p}}$ is a unit and, by Lemma 3.3. $(\tilde{h}+\tilde{f})_{-}$is also a unit in $\mathcal{S}^{*}(M)_{\mathfrak{p}}$. This contradicts the fact that $(\tilde{h}+\tilde{f})_{-} \in \mathfrak{p}$. Therefore, $\varphi^{\star}$ satisfies the going-up property.

Corollary 3.5. Let $\varphi: N \rightarrow M$ be an open, closed and surjective semialgebraic map. Let $\mathfrak{q} \in \operatorname{Spec}_{\mathrm{S}}^{*}(N)$ and $\mathfrak{p}=\mathfrak{q} \cap \mathcal{S}^{*}(M)$. Then, the ring homomorphism $\psi$ : $\mathcal{S}^{*}(M)_{\mathfrak{p}} \rightarrow \mathcal{S}^{*}(N)_{\mathfrak{q}}$ induced by $\varphi$ satisfies the going-up property and it is injective.

Proof. First, let us check the going-up property. Indeed, let $\mathfrak{q}_{1}$ be a prime ideal in $\mathcal{S}^{*}(N)$ with $\mathfrak{q}_{1} \subsetneq \mathfrak{q}$ such that $\mathfrak{p}_{1}=\mathfrak{q}_{1} \cap \mathcal{S}^{*}(M) \subsetneq \mathfrak{p}$. Consider a prime ideal $\mathfrak{p}_{2}$ in $\mathcal{S}^{*}(M)$ such that $\mathfrak{p}_{1} \subsetneq \mathfrak{p}_{2} \subsetneq \mathfrak{p}$. By Lemma 3.4 there exists $\mathfrak{q}_{2} \in \operatorname{Spec}_{\mathrm{s}}^{*}(N)$ such that $\mathfrak{q}_{1} \subsetneq \mathfrak{q}_{2}$ and $\mathfrak{p}_{2}=\mathfrak{q}_{2} \cap \mathcal{S}^{*}(M)$. It only remains to check that $\mathfrak{q}_{2} \in \operatorname{Spec}\left(\mathcal{S}^{*}(N)_{\mathfrak{q}}\right)$, that is, $\mathfrak{q}_{2} \subset \mathfrak{q}$. But since the set of prime ideals of $\mathcal{S}^{*}(N)$ containing $\mathfrak{q}_{1}$ is, by (2.13), a chain and $\mathfrak{q}_{2} \cap \mathcal{S}^{*}(M)=\mathfrak{p}_{2} \subsetneq \mathfrak{p}=\mathfrak{q} \cap \mathcal{S}^{*}(M)$, we conclude $\mathfrak{q}_{2} \subsetneq \mathfrak{q}$.

Next, let us show the injectivity of $\psi$. Let $F \in \mathcal{S}^{*}(M)$ and $f \in \mathcal{S}^{*}(M) \backslash \mathfrak{p}$ be such that $F / f \in \operatorname{ker} \psi$. Thus, $g F=0$ for some $g \in \mathcal{S}^{*}(N) \backslash \mathfrak{q}$ and, after changing $g$ by $|g|$ if necessary, we may assume that $g$ is nonnegative. Clearly, $0 \leq g \leq g_{+} \circ \varphi$ on $N$; hence, by (2.12),$g_{+} \circ \varphi \notin \mathfrak{q}$, and so $g_{+} \notin \mathfrak{p}$. Consequently, to prove the injectivity of $\psi$, it suffices to check that $g_{+} F=0$. Otherwise, there would exist a point $y \in M$ such that $g_{+}(y) F(y) \neq 0$; in particular, $F(y) \neq 0$. Therefore, for each $x \in \varphi^{-1}(y)$,

$$
0=(g(F \circ \varphi))(x)=g(x) F(\varphi(x))=g(x) F(y),
$$

and so $g(x)=0$. Thus, $\varphi^{-1}(y) \subset Z_{N}(g)$, and this implies $g_{+}(y)=0$, a contradiction.

Lemma 3.6 (Going-down). Let $\varphi: N \rightarrow M$ be an open, closed and surjective semialgebraic map. Then, the homomorphism $\varphi^{\star}: \mathcal{S}^{*}(M) \rightarrow \mathcal{S}^{*}(N), f \mapsto f \circ \varphi$ satisfies the going-down property.

Proof. Let $\mathfrak{q}$ be a prime ideal in $\mathcal{S}^{*}(N)$ and let $\mathfrak{p}=\mathfrak{q} \cap \mathcal{S}^{*}(M)$. We must prove that the induced map $\Phi: \operatorname{Spec}\left(\mathcal{S}^{*}(N)_{\mathfrak{q}}\right) \rightarrow \operatorname{Spec}\left(\mathcal{S}^{*}(M)_{\mathfrak{p}}\right)$ is surjective. It is a closed map, by Corollary [3.5 and [AM, §5], and so its image im $\Phi$ is a closed subset of $\operatorname{Spec}\left(\mathcal{S}^{*}(M)_{\mathfrak{p}}\right)$. Therefore, it is enough to see that im $\Phi$ is a dense subspace of $\operatorname{Spec}\left(\mathcal{S}^{*}(M)_{\mathfrak{p}}\right)$. 
To that end we will use the injectivity of $\psi: \mathcal{S}^{*}(M)_{\mathfrak{p}} \rightarrow \mathcal{S}^{*}(N)_{\mathfrak{q}}$ proved in Corollary 3.5 to show that im $\Phi$ contains the set of minimal prime ideals of $\mathcal{S}^{*}(M)_{\mathfrak{p}}$, which is a dense subset of $\operatorname{Spec}\left(\mathcal{S}^{*}(M)_{\mathfrak{p}}\right)$ because the ring $\mathcal{S}^{*}(M)_{\mathfrak{p}}$ has finite Krull dimension; see [FG1, 4.1].

Indeed, to simplify notation write $A=\mathcal{S}^{*}(M)_{\mathfrak{p}}$ and $B=\mathcal{S}^{*}(N)_{\mathfrak{q}}$. Given a minimal prime ideal $\mathfrak{a}$ in $A$, the induced homomorphism $\psi_{\mathfrak{a}}: A_{\mathfrak{a}} \rightarrow B_{\mathfrak{a}}$ is also injective. Whence $B_{\mathfrak{a}}$ is not zero and so it has a maximal ideal $\mathfrak{b}$. Thus, $\psi_{\mathfrak{a}}^{-1}(\mathfrak{b})$ is a prime ideal in $A_{\mathfrak{a}}$ which must be $\mathfrak{a} A_{\mathfrak{a}}$ (because it is the unique prime ideal of $\left.A_{\mathfrak{a}}\right)$; hence, $\Phi(\mathfrak{b})=\mathfrak{a}$.

The next lemma, which will be used later, reduces the proof of Theorem 1.5 to studying the behaviour of the spectral map between the Zariski spectra. More precisely,

Lemma 3.7. Let $\varphi: N \rightarrow M$ be a semialgebraic map such that the induced map $\operatorname{Spec}_{\mathrm{S}}^{\diamond}(\varphi): \operatorname{Spec}_{\mathrm{S}}^{\diamond}(N) \rightarrow \operatorname{Spec}_{\mathrm{s}}^{\diamond}(M)$ is open, closed and surjective. Then, $\beta_{\mathrm{s}}^{\diamond} \varphi:$ $\beta_{\mathrm{s}}^{\diamond} N \rightarrow \beta_{\mathrm{s}}^{\diamond} M$ is an open, proper and surjective map.

Proof. Since $\operatorname{Spec}_{\mathrm{s}}^{\diamond}(\varphi): \operatorname{Spec}_{\mathrm{s}}^{\diamond}(N) \rightarrow \operatorname{Spec}_{\mathrm{s}}^{\diamond}(M)$ is closed, it maps closed points to closed points and therefore it restricts to $\beta_{\mathrm{s}}^{\diamond} N$ as $\beta_{\mathrm{s}}^{\diamond} \varphi: \beta_{\mathrm{s}}^{\diamond} N \rightarrow \beta_{\mathrm{s}}^{\diamond} M$. Moreover, since $\beta_{\mathrm{s}}^{\diamond} \varphi$ is continuous, $\beta_{\mathrm{s}}^{\diamond} N$ is a compact space and $\beta_{\mathrm{s}}^{\diamond} M$ is Hausdorff, it follows

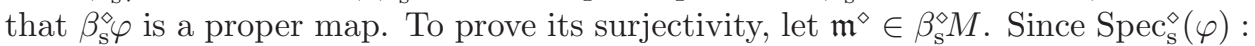
$\operatorname{Spec}_{\mathrm{s}}^{\diamond}(N) \rightarrow \operatorname{Spec}_{\mathrm{s}}^{\diamond}(M)$ is surjective, there exists a prime ideal $\mathfrak{p} \in \operatorname{Spec}_{\mathrm{s}}^{\diamond}(N)$ with $\operatorname{Spec}_{\mathbb{S}}^{\diamond}(\varphi)(\mathfrak{p})=\mathfrak{m}^{\diamond}$. Let $\mathfrak{n}^{\diamond}$ be (the unique) maximal ideal of $\mathcal{S}^{\diamond}(N)$ containing $\mathfrak{p}$. Then $\mathfrak{m}^{\diamond}=\operatorname{Spec}_{\mathrm{s}}^{*}(\varphi)(\mathfrak{p}) \subset \operatorname{Spec}_{\mathrm{s}}^{*}(\varphi)\left(\mathfrak{n}^{\diamond}\right)$ and, $\mathfrak{m}^{\diamond}$ being a maximal ideal of $\mathcal{S}^{\diamond}(M)$ and $\beta_{\mathrm{s}}^{\diamond} \varphi=\left.\operatorname{Spec}_{\mathrm{s}}^{\diamond}(\varphi)\right|_{\beta_{\mathrm{s}}^{\diamond} N}$, we get $\mathfrak{m}^{\diamond}=\beta_{\mathrm{s}}^{\diamond} \varphi\left(\mathfrak{n}^{\diamond}\right)$.

As to the openness of $\beta_{\mathrm{s}}^{\diamond} \varphi$, let $W$ be an open subset of $\beta_{\mathrm{s}}^{\diamond} N$, and denote by $\rho_{M}$ : $\operatorname{Spec}_{\mathrm{s}}^{\diamond}(M) \rightarrow \beta_{\mathrm{s}}^{\diamond} M$ the retraction that maps each prime ideal of $\mathcal{S}^{\diamond}(M)$ to the unique maximal ideal of $\mathcal{S}^{\diamond}(M)$ containing it. The equality $\beta_{\mathrm{s}}^{\diamond} \varphi(W)=\operatorname{Spec}_{\mathrm{s}}^{\diamond}(\varphi)\left(\rho_{M}^{-1}(W)\right) \cap$ $\beta_{\mathrm{s}}^{\diamond} M$ follows readily, and it proves that $\beta_{\mathrm{s}}^{\diamond} \varphi(W)$ is an open subset of $\beta_{\mathrm{s}}^{\diamond} M$, because $\rho_{M}$ is continuous and $\operatorname{Spec}_{\mathrm{S}}^{\diamond}(\varphi)$ is an open map.

Lemma 3.8. Let $\varphi: N \rightarrow M$ be a semialgebraic map. Then, the fibers of the spectral map $\operatorname{Spec}_{\mathrm{S}}^{\diamond}(\varphi): \operatorname{Spec}_{\mathrm{S}}^{\diamond}(N) \rightarrow \operatorname{Spec}_{\mathrm{S}}^{\diamond}(M)$ are compact.

Proof. Let $\mathfrak{p}$ be a prime ideal of $\mathcal{S}^{\diamond}(M)$ and write $K=\operatorname{Spec}_{\mathrm{s}}^{\diamond}(\varphi)^{-1}(\mathfrak{p})$. The closure

$$
\Gamma=\mathrm{Cl}_{\operatorname{Spec}_{\mathbf{s}}^{\diamond}(M)}(\{\mathfrak{p}\})
$$

of $\{\mathfrak{p}\}$ is a finite and totally ordered set $\Gamma=\left\{\mathfrak{p}, \mathfrak{p}_{1}, \ldots, \mathfrak{p}_{r}\right\}$, with $\mathfrak{p} \subsetneq \mathfrak{p}_{1} \subsetneq \ldots \subsetneq \mathfrak{p}_{r}$. Now we distinguish two cases. If $\mathfrak{p}$ is maximal, then $K=\operatorname{Spec}_{\mathrm{s}}^{\diamond}(\varphi)^{-1}(\Gamma)$ is a closed subset of the compact space $\operatorname{Spec}_{\mathrm{s}}^{\diamond}(N)$; hence it is compact.

If $\mathfrak{p}$ is not maximal, let us choose a function $f \in \mathfrak{p}_{1} \backslash \mathfrak{p}$. Then,

$$
K=\operatorname{Spec}_{\mathrm{s}}^{\diamond}(\varphi)^{-1}\left(\Gamma \cap \mathcal{D}_{\operatorname{Spec}_{\mathrm{s}}^{\diamond}(M)}(f)\right)=\operatorname{Spec}_{\mathrm{s}}^{\diamond}(\varphi)^{-1}(\Gamma) \cap \mathcal{D}_{\operatorname{Spec}_{\mathrm{s}}^{\diamond}(N)}(f \circ \varphi)
$$

is compact because it is a closed subset of the compact set $\mathcal{D}_{\operatorname{Spec}_{\AA}^{\diamond}(N)}(f \circ \varphi)$.

Now, we are ready to prove Theorem 1.5

Proof of Theorem 1.5. First, observe that by Lemma 3.7, it is enough to prove that $\operatorname{Spec}_{\mathrm{s}}^{*}(\varphi): \operatorname{Spec}_{\mathrm{s}}^{*}(N) \rightarrow \operatorname{Spec}_{\mathrm{s}}^{*}(M)$ is open, proper and surjective. Indeed, the closedness of $\operatorname{Spec}_{\mathrm{S}}^{*}(\varphi)$ is a straightforward consequence of the going-up property (see [AM, §5]) proved in Lemma 3.4. while its surjectivity follows because the image 
of $\operatorname{Spec}_{\mathrm{s}}^{*}(\varphi)$ is closed and it contains the dense subset $\varphi(N)=M \equiv\left\{\mathfrak{m}_{y}^{*}: y \in M\right\}$ of $\operatorname{Spec}_{\mathrm{S}}^{*}(M)$. Moreover, by Lemma 3.8, the fibers of $\operatorname{Spec}_{\mathrm{s}}^{*}(\varphi)$ are compact. Thus, it just remains to check that $\operatorname{Spec}_{\mathrm{S}}^{*}(\varphi)$ is an open map. For that, it is enough to check the equality

$$
\mathcal{D}_{\operatorname{Spec}_{\mathrm{s}}^{*}(M)}\left(h_{+}\right)=\operatorname{Spec}_{\mathrm{s}}^{*}(\varphi)\left(\mathcal{D}_{\operatorname{Spec}_{\mathrm{s}}^{*}(N)}(h)\right)
$$

for every nonnegative function $h \in \mathcal{S}^{*}(N)$, because $\mathcal{D}_{\operatorname{Spec}_{\mathrm{s}}^{*}(N)}(|h|)=\mathcal{D}_{\operatorname{Spec}_{\mathrm{s}}^{*}(N)}(h)$.

Notice that $0 \leq h \leq h_{+} \circ \varphi$, which implies, by (2.112), that $h_{+} \notin \operatorname{Spec}_{\mathrm{s}}^{*}(\varphi)(\mathfrak{q})$ whenever $h \notin \mathfrak{q}$. Conversely, let $\mathfrak{p} \notin \operatorname{Spec}_{\mathrm{S}}^{*}(\varphi)\left(\mathcal{D}_{\operatorname{Spec}_{\mathrm{s}}^{*}(N)}(h)\right)$ be a prime ideal of $\mathcal{S}^{*}(M)$ and let us prove that $h_{+} \in \mathfrak{p}$. Indeed, denote $\mathfrak{a}=\mathfrak{p} \mathcal{S}^{*}(N)$ and let us show first that $h \in \sqrt{\mathfrak{a}}=\bigcap_{\mathfrak{q}} \mathfrak{q}$ where $\mathfrak{q} \in \mathcal{Z}_{\operatorname{Spec}_{\mathrm{s}}^{*}(N)}(\mathfrak{a})$.

Otherwise, there would exist a prime ideal $\mathfrak{q}_{1}$ in $\mathcal{S}^{*}(N)$ containing a such that $h \notin \mathfrak{q}_{1}$. The prime ideal $\mathfrak{p}_{1}=\mathfrak{q}_{1} \cap \mathcal{S}^{*}(M)$ contains $\mathfrak{p}$ and, by the going-down property of Lemma 3.6. there exists a prime ideal $\mathfrak{q}$ in $\mathcal{S}^{*}(N)$ lying over $\mathfrak{p}$ and contained in $\mathfrak{q}_{1}$. Consequently, $h \notin \mathfrak{q} \in\left(\operatorname{Spec}_{\mathrm{s}}^{*}(\varphi)\right)^{-1}(\mathfrak{p}) \subset z_{\operatorname{Spec}_{\mathrm{s}}^{*}(N)}(h)$, because $\mathfrak{p} \notin \operatorname{Spec}_{\mathrm{s}}^{*}(\varphi)\left(\mathcal{D}_{\operatorname{Spec}_{\mathrm{s}}^{*}(N)}(h)\right)$, a contradiction.

Hence, some power of $h$ occurs in $\mathfrak{a}$; that is, $h^{\ell}=\sum_{i=1}^{d} f_{i} g_{i}$ for some $\ell \geq 1$, $f_{i} \in \mathfrak{p}$ and $g_{i} \in \mathcal{S}^{*}(N)$. Let $F_{i}=\left|f_{i}\right| \in \mathfrak{p}$ and $G_{i}=\left|g_{i}\right| \in \mathcal{S}^{*}(N)$; hence, $h^{\ell} \leq \sum_{i=1}^{d}\left(F_{i} \circ \varphi\right) G_{i}$ on $N$ and so $0 \leq\left(h^{\ell}\right)_{+} \leq \sum_{i=1}^{d}\left(G_{i}\right)_{+} F_{i}$ on $M$. By (2.112), this implies, since $\sum_{i=1}^{d} G_{i,+} F_{i} \in \mathfrak{p}$, that also $h_{+}^{\ell} \in \mathfrak{p}$, that is, $h_{+} \in \mathfrak{p}$, as wanted.

Our next goal is to approach Theorem [1.6, Before that we need some preliminaries:

Remarks 3.9. (i) To study the map $\operatorname{Spec}_{\mathrm{S}}(\varphi): \operatorname{Spec}_{\mathrm{s}}(N) \rightarrow \operatorname{Spec}_{\mathrm{s}}(M)$ induced by a semialgebraic map $\varphi: N \rightarrow M$ by using similar arguments to those used for the spectra of rings of bounded semialgebraic functions, it is natural to impose $\varphi$ to be a proper and surjective map. Under this assumption its fibers are nonempty compact sets and so each function $h \in \mathcal{S}(N)$ induces two functions:

$$
h_{+}: M \rightarrow \mathbb{R}, y \mapsto \max \{h(x): \varphi(x)=y\}
$$

and

$$
h_{-}: M \rightarrow \mathbb{R}, y \mapsto \min \{h(x): \varphi(x)=y\} .
$$

Moreover, an analogous proof to the one of Construction 3.1 shows that, if $\varphi$ is also an open map, then both $h_{+}$and $h_{-}$are semialgebraic functions; that is, they are continuous with semialgebraic graphs.

(ii) On the other hand, as is well known, $\operatorname{Spec}_{\mathrm{s}}(N)$ and $\operatorname{Spec}_{\mathrm{s}}(M)$ are homeomorphic to the subsets $\mathfrak{S}(N)$ and $\mathfrak{S}(M)$ of $\operatorname{Spec}_{\mathrm{s}}^{*}(N)$ and $\operatorname{Spec}_{\mathrm{s}}^{*}(M)$, respectively, defined by

$$
\mathfrak{S}(N)=\left\{\mathfrak{p} \in \operatorname{Spec}_{\mathrm{S}}^{*}(N): \mathfrak{p} \cap \mathcal{W}(N)=\varnothing\right\}
$$

and

$$
\mathfrak{S}(M)=\left\{\mathfrak{q} \in \operatorname{Spec}_{\mathrm{s}}^{*}(M): \mathfrak{q} \cap \mathcal{W}(M)=\varnothing\right\},
$$


where $\mathcal{W}(N)=\left\{f \in \mathcal{S}^{*}(N): Z_{N}(f)=\varnothing\right\}$ and $\mathcal{W}(M)=\left\{g \in \mathcal{S}^{*}(M): Z_{M}(g)=\right.$ $\varnothing\}$ (see for instance [FG2, 3.2]). After identifying these spaces, we get a commutative diagram whose horizontal arrows are embeddings:

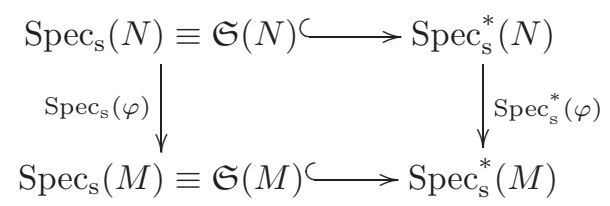

(iii) Again, the homomorphism $\varphi^{\star}: \mathcal{S}(M) \rightarrow \mathcal{S}(N), f \mapsto f \circ \varphi$ endows $\mathcal{S}(N)$ with a structure of $\mathcal{S}(M)$-module, and in fact $\mathcal{S}(N)$ is the ring of fractions of $\mathcal{S}^{*}(N)$ with respect to $\mathcal{W}(M)$. To prove this, it suffices to write each function $f \in \mathcal{S}(N)$ as a quotient $f=g / h$ where $g=f /\left(1+f_{+}^{2}\right) \in \mathcal{S}^{*}(N)$ and $h=1 /\left(1+f_{+}^{2}\right) \in \mathcal{W}(M)$. In other words, the canonical homomorphism $\mathcal{S}^{*}(N)_{\mathcal{W}(M)} \rightarrow \mathcal{S}^{*}(N)_{\mathcal{W}(N)}=\mathcal{S}(N)$ is an isomorphism.

(iv) Hence, $\mathfrak{S}(N)=\left\{\mathfrak{p} \in \operatorname{Spec} \mathcal{S}^{*}(N): \mathfrak{p} \cap \mathcal{W}(M)=\varnothing\right\}$, and so

$$
\operatorname{Spec}_{\mathrm{s}}^{*}(\varphi)\left(\operatorname{Spec}_{\mathrm{s}}^{*}(N) \backslash \operatorname{Spec}_{\mathrm{s}}(N)\right)=\operatorname{Spec}_{\mathrm{s}}^{*}(M) \backslash \operatorname{Spec}_{\mathrm{s}}(M) .
$$

Therefore, $\operatorname{Spec}_{\mathrm{s}}(N)=\operatorname{Spec}_{\mathrm{s}}^{*}(\varphi)^{-1}\left(\operatorname{Spec}_{\mathrm{s}}(M)\right)$.

For the sake of the reader we state next a useful auxiliary result whose proof can be found in [Fe2, 3.9].

Lemma 3.10. Let $\varphi: N \rightarrow M$ be a surjective semialgebraic map. Then, $\varphi$ is proper if and only if $\beta_{\mathrm{s}}^{*} \varphi(\partial N)=\partial M$.

We are now in a position to prove Theorem 1.6. Namely,

Proof of Theorem 1.6. We will prove the chain of implications (i) $\Longrightarrow$ (ii) $\Longrightarrow$ (iii) $\Longrightarrow$ (iv) $\Longrightarrow$ (i). Observe first that (i) $\Longrightarrow$ (ii) follows from Theorem 1.5 and Lemma 3.10 .

(ii) $\Longrightarrow$ (iii) By Remark [3.9(iv), we have a commutative diagram

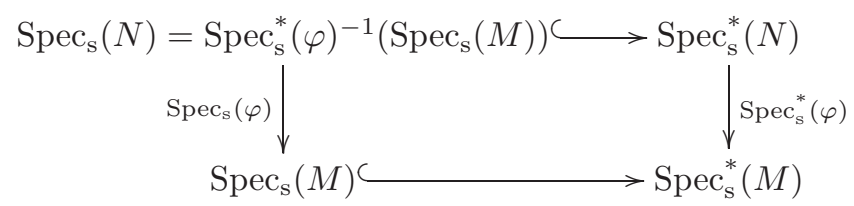

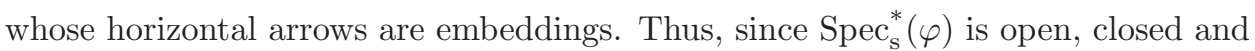
surjective, so is $\operatorname{Spec}_{\mathrm{s}}(\varphi)$. Moreover, by Lemma 3.8 the fibers of $\operatorname{Spec}_{\mathrm{s}}(\varphi)$ are compact, and so $\operatorname{Spec}_{\mathrm{S}}(\varphi): \operatorname{Spec}_{\mathrm{S}}(N) \rightarrow \operatorname{Spec}_{\mathrm{s}}(M)$ is also a proper map. Next, the equality $\beta_{\mathrm{s}}^{*} \varphi(\partial N)=\partial M$ implies that $\varphi^{-1}(p)=\left(\beta_{\mathrm{s}}^{*} \varphi\right)^{-1}\left(\mathfrak{m}_{p}^{*}\right)$, and this last set is a compact subset of $N$ for all $p \in M$, in particular, if $p$ is an isolated point of $M$.

(iii) $\Longrightarrow$ (iv). It follows from Lemma 3.7 that $\beta_{\mathrm{s}} \varphi: \beta_{\mathrm{s}} N \rightarrow \beta_{\mathrm{s}} M$ is an open, proper and surjective map. Thus, from (1.1)-(1.4), the same holds true for the map $\beta_{\mathrm{s}}^{*} \varphi: \beta_{\mathrm{s}}^{*} N \rightarrow \beta_{\mathrm{s}}^{*} M$, and it only remains to check that $\beta_{\mathrm{s}}^{*} \varphi(\partial N)=\partial M$. This last task will be done in several steps. In any case, we may assume from the beginning that $N \subset \mathbb{R}^{n}$ is bounded, after changing $N$ by its inverse image under the semialgebraic homeomorphism

$$
\mathbb{B}_{n}(0,1) \rightarrow \mathbb{R}^{n}, x \mapsto \frac{x}{\sqrt{1-\|x\|^{2}}}
$$


between the open ball $\mathbb{B}_{n}(0,1) \subset \mathbb{R}^{n}$ of center the origin and radius 1 and $\mathbb{R}^{n}$.

(1.6.1) The fiber $\varphi^{-1}(p)$ is nowhere dense in $N$ for each nonisolated point $p$ of $M$.

Suppose that there exist a nonisolated point $p \in M$ and a nonempty semialgebraic subset $W \subset \varphi^{-1}(p)$ which is, moreover, open in $N$. Let us check first that $V=W \backslash \mathrm{Cl}_{\beta_{\mathrm{s}}^{*} N}(\partial N)$ is a nonempty open subset of $\beta_{\mathrm{s}}^{*} N$. Indeed, let $N_{\mathrm{lc}}$ be the largest locally compact and dense subset of $N$, and let $\rho_{1}(N)=N \backslash N_{\mathrm{lc}}=\mathrm{Cl}_{\mathbb{R}^{n}}\left(\mathrm{Cl}_{\mathbb{R}^{n}}(N) \backslash\right.$ $N) \cap N$ (see [Fe1, 3.8]). By [Fe1, 6.8.1], $\mathrm{Cl}_{\beta_{\mathrm{s}}^{*} N}(\partial N)=\beta_{\mathrm{s}}^{*} N \backslash N_{\mathrm{lc}}$. Notice that by its definition $\rho_{1}(N) \cap W=\rho_{1}(W)$; hence $W_{\mathrm{lc}}=W \backslash \rho_{1}(W)=W \backslash \rho_{1}(N) \neq \varnothing$. Thus,

$$
V=W \backslash\left(\beta_{\mathrm{s}}^{*} N \backslash N_{\mathrm{lc}}\right)=W \backslash\left(N \backslash N_{\mathrm{lc}}\right)=W \backslash \rho_{1}(N) \neq \varnothing .
$$

Moreover, $V \subset N_{\text {lc }} \subset N$, and so $V$ is an open subset of $N_{\text {lc }}$; hence $V$ is an open subset of $\beta_{\mathrm{s}}^{*} N$, because the locally compact semialgebraic set $N_{\mathrm{lc}}$ is open in its Hausdorff compactification $\beta_{\mathrm{s}}^{*} N$. Now, $\beta_{\mathrm{s}}^{*} \varphi: \beta_{\mathrm{s}}^{*} N \rightarrow \beta_{\mathrm{s}}^{*} M$ being an open map, $\beta_{\mathrm{s}}^{*} \varphi(V)=\varphi(V)=\{p\}$ is an open subset of $M$, which contradicts the fact that $p$ is nonisolated in $M$.

(1.6) 2) The fiber $\varphi^{-1}(p)$ is a compact set for all $p \in M$.

Fix a point $p \in M$; since by hypothesis $\varphi^{-1}(p)$ is compact for each isolated point $p$ of $M$, we may assume that $p$ is a nonisolated point. Suppose, by way of contradiction, that $C=\varphi^{-1}(p)$ is not compact and let $q \in \mathrm{Cl}_{\mathbb{R}^{n}}(C) \backslash C$. Since $C$ is, by 1.6]1, nowhere dense in $N$, we have $q \in \mathrm{Cl}_{\mathbb{R}^{n}}(C) \subset \mathrm{Cl}_{\mathbb{R}^{n}}(N)=\mathrm{Cl}_{\mathbb{R}^{n}}(N \backslash C)$. By the Curve Selection Lemma [BCR, 2.5.5], there exists a semialgebraic path $\gamma:[0,1] \rightarrow \mathrm{Cl}_{\mathbb{R}^{n}}(N)$ such that $\gamma((0,1]) \subset N \backslash C$ and $\gamma(0)=q$. Consider the maximal ideal

$$
\mathfrak{n}_{\gamma}=\left\{f \in \mathcal{S}(N): \exists \varepsilon>0 \text { such that }\left.(f \circ \gamma)\right|_{(0, \varepsilon]}=0\right\}
$$

of $\mathcal{S}(N)$ (see [Fe2, 3.4]). The image of $\mathfrak{n}_{\gamma}$ under $\operatorname{Spec}_{\mathbf{s}}(\varphi)$ is a maximal ideal, because $\operatorname{Spec}_{\mathrm{s}}(\varphi)$ maps closed points into closed points. Thus $\operatorname{Spec}_{\mathbf{s}}(\varphi)\left(\mathfrak{n}_{\gamma}\right)=\mathfrak{m}_{p}$, by the choice of $\gamma$. However, the semialgebraic function $f=\|x-p\| \in \mathfrak{m}_{p} \backslash \operatorname{Spec}_{\mathrm{s}}(\varphi)\left(\mathfrak{n}_{\gamma}\right)$ because the composition $(f \circ \varphi \circ \gamma)(t)=\|(\varphi \circ \gamma)(t)-p\|$ does not vanish identically on any interval of the type $(0, \varepsilon]$ with $\varepsilon>0$, since $\gamma((0,1]) \subset N \backslash C=N \backslash \varphi^{-1}(p)$.

[1.6]3) $\beta_{\mathrm{s}}^{*} \varphi(\partial N) \cap M=\varnothing$, or equivalently, $\beta_{\mathrm{s}}^{*} \varphi(\partial N)=\partial M$.

Suppose there exists a point $p \in \beta_{\mathrm{s}}^{*} \varphi(\partial N) \cap M$. Then, there exists $\mathfrak{n} \in \partial N \subset$ $\beta_{\mathrm{s}}^{*} N \subset \operatorname{Spec}_{\mathrm{s}}^{*}(N) \operatorname{such}$ that $\operatorname{Spec}_{\mathrm{s}}^{*}(\varphi)(\mathfrak{n})=\beta_{\mathrm{s}}^{*} \varphi(\mathfrak{n})=\mathfrak{m}_{p}$. By [FG4, 5.4], we may assume that

$$
\mathfrak{n}=\mathfrak{n}_{\alpha}=\left\{f \in \mathcal{S}(N): \exists \varepsilon>0 \text { such that }\left.(f \circ \alpha)\right|_{(0, \varepsilon]}=0\right\}
$$

for some semialgebraic path $\alpha:[0,1] \rightarrow \mathrm{Cl}_{\mathbb{R}^{n}}(N)$ such that $\alpha((0,1]) \subset N$ and $q=\alpha(0) \in \mathrm{Cl}_{\mathbb{R}^{n}}(N) \backslash N$. Since the semialgebraic function $f=\|x-p\| \in \mathfrak{m}_{p}=$ $\operatorname{Spec}_{\mathrm{s}}^{*}(\varphi)\left(\mathfrak{n}_{\alpha}\right)$, there exists $\varepsilon>0$ such that $\left.(f \circ \varphi \circ \alpha)\right|_{(0, \varepsilon]}=\|(\varphi \circ \alpha)(t)-p\| \|_{(0, \varepsilon]} \equiv 0$. We may assume that $\varepsilon=1$, that is, $(\varphi \circ \gamma)(t)=p$ for all $t \in[0,1]$. Hence, $\alpha((0,1]) \subset \varphi^{-1}(p)$, and since $\varphi^{-1}(p)$ is compact, $q=\alpha(0) \in \varphi^{-1}(p) \subset N$, a contradiction. Thus, $\beta_{\mathrm{s}}^{*} \varphi(\partial N) \cap M=\varnothing$, as wanted.

(iv) $\Longrightarrow$ (i). Since $\beta_{\mathrm{s}}^{*} \varphi: \beta_{\mathrm{s}}^{*} N \rightarrow \beta_{\mathrm{s}}^{*} M$ is surjective and $\beta_{\mathrm{s}}^{*} \varphi(\partial N)=\partial M$, the map $\varphi$ is surjective and, by Lemma 3.10, it is also proper. Finally, given an open subset 
$W$ of $\beta_{\mathrm{s}}^{*} N$, and since $\beta_{\mathrm{s}}^{*} \varphi(\partial N)=\partial M$, we have

$$
\begin{aligned}
\beta_{\mathrm{s}}^{*} \varphi(W) \cap M & =\left(\beta_{\mathrm{s}}^{*} \varphi(W \cap N) \cap M\right) \cup\left(\beta_{\mathrm{s}}^{*} \varphi(W \cap \partial N) \cap M\right) \\
& =\beta_{\mathrm{s}}^{*} \varphi(W \cap N) \cap M=\varphi(W \cap N),
\end{aligned}
$$

which implies that $\varphi$ is an open map, because $\beta_{\mathrm{s}}^{*} \varphi$ is.

Remarks 3.11. (i) Assertions (ii) and (iv) in Theorem 1.6 are not equivalent to assertion (i) if we eliminate the hypothesis $\beta_{\mathrm{s}}^{*} \varphi(\partial N)=\partial M$. Indeed, the inclusion map $\varphi:(0,1) \hookrightarrow[0,1]$ is neither proper nor closed nor surjective, but the induced maps

$$
\begin{aligned}
& \operatorname{Spec}_{\mathrm{s}}^{*}(\varphi)=\mathrm{id}: \operatorname{Spec}_{\mathrm{s}}^{*}((0,1))=\operatorname{Spec}_{\mathrm{s}}^{*}([0,1]) \rightarrow \operatorname{Spec}_{\mathrm{s}}^{*}([0,1]), \text { and } \\
& \beta_{\mathrm{s}}^{*} \varphi=\mathrm{id}: \beta_{\mathrm{s}}^{*}(0,1)=\beta_{\mathrm{s}}^{*}[0,1]=[0,1] \rightarrow[0,1]
\end{aligned}
$$

are open, proper and surjective. Indeed, see [FG3, 3.9], the rings $\mathcal{S}^{*}((0,1))$ and

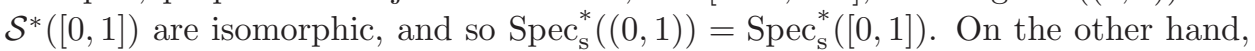
for the equalities $\beta_{\mathrm{s}}^{*}(0,1)=\beta_{\mathrm{s}}^{*}[0,1]=[0,1]$ see [FG4, 4.9].

(ii) Assertion (iii) in Theorem 1.6 is not equivalent to assertion (i) if we eliminate the hypothesis that the fiber under $\varphi$ of each isolated point of $M$ is compact. Indeed, if $\varphi: N \rightarrow\{p\}$ is a constant semialgebraic map and $N$ is not compact, the map $\operatorname{Spec}_{\mathrm{s}}(\varphi): \operatorname{Spec}_{\mathrm{s}}(N) \rightarrow \operatorname{Spec}_{\mathrm{s}}(\{p\}) \equiv\{p\}$ is open, proper and surjective, while $\varphi$ is not proper.

\section{REFERENCES}

[AM] M.F. Atiyah, I.G. Macdonald: Introduction to commutative algebra. Addison-Wesley Publishing Co., Reading, Mass.-London-Don Mills, Ontario: 1969. MR0242802 (39:4129)

[BCR] J. Bochnak, M. Coste, M.-F. Roy: Real algebraic geometry. Ergeb. Math. 36, SpringerVerlag, Berlin: 1998. MR1659509 (2000a:14067)

[B] G.W. Brumfiel: Quotient spaces for semialgebraic equivalence relations. Math. Z. 195 (1987), no. 1, 69-78. MR888127 (88i:14015)

[Fe1] J.F. Fernando: On chains of prime ideals in rings of semialgebraic functions. Preprint $R A A G$ (2010). http://www.mat.ucm.es/ josefer/pdfs/preprint/chains.pdf

$[\mathrm{Fe} 2]$ J.F. Fernando: On distinguished points of the remainder of the semialgebraic Stone-Čech compactification of a semialgebraic set. Preprint RAAG (2010). http://www. mat.ucm.es/ josefer/pdfs/preprint/remainder.pdf

[FG1] J.F. Fernando, J.M. Gamboa: On the Krull dimension of rings of semialgebraic functions. Preprint RAAG (2010). http://www.mat.ucm.es/ josefer/pdfs/preprint/dim.pdf

[FG2] J.F. Fernando, J.M. Gamboa: On the spectra of rings of semialgebraic functions. Collectanea Mathematica, to appear. http://www.mat.ucm.es/ josefer/pdfs/ preprint/spectra.pdf

[FG3] J.F. Fernando, J.M. Gamboa: On Banach-Stone type theorems for semialgebraic sets. Preprint RAAG (2010). http://www.mat.ucm.es/ josefer/pdfs/preprint/homeo.pdf

[FG4] J.F. Fernando, J.M. Gamboa: On the semialgebraic Stone-Čech compactification of a semialgebraic set. Trans. Amer. Math. Soc. (to appear). http://www.mat.ucm.es/ $\sim$ josefer/pdfs/preprint/mspectra.pdf

[MO] G. De Marco, A. Orsatti: Commutative rings in which every prime ideal is contained in a unique maximal ideal. Proc. Amer. Math. Soc. 30 (1971), no. 3, 459-466. MR0282962 $(44: 196)$

[Mu] M.-A. Mulero: Algebraic properties of rings of continuous functions. Fund. Math. 149 (1996), no. 1, 55-66. MR1372357(97c:16038) 
[P] V.I. Ponomarev: Open mappings of normal spaces. Dokl. Akad. Nauk SSSR 126 (1959), 716-718. MR0107855 (21:6577)

[PS] C. Procesi, G. Schwarz: Inequalities defining orbit spaces. Invent. Math. 81 (1985), no. 3, 539-554. MR807071 (87h:20078)

Departamento de Álgebra, Facultad de Ciencias Matemáticas, Universidad ComPlutense de Madrid, 28040 Madrid, Spain

E-mail address: josefer@mat.ucm.es

Departamento de Álgebra, Facultad de Ciencias Matemáticas, Universidad ComPlutense De Madrid, 28040 Madrid, Spain

E-mail address: jmgamboa@mat.ucm.es 